\title{
W-Band Characterization of Grounded Frequency Selective Surface Arrays Composed of Nonequal Slot Length Subarrays
}

\author{
S. Islam, ${ }^{1}$ J. Stiens, ${ }^{1}$ G. Poesen, ${ }^{1}$ I. Jaeger, ${ }^{1}$ W. De Raedt, ${ }^{2}$ and R. Vounckx ${ }^{1}$ \\ ${ }^{1}$ Laboratory for Micro- and Photonelectronics, Department of Electronics and Informatics, Vrije Universiteit Brussel, \\ Pleinlaan 2, 1050 Brussels, Belgium \\ ${ }^{2}$ Department INTEGSYS, IMEC, Kapeldreef 75, 3001 Heverlee, Belgium
}

Correspondence should be addressed to S. Islam, sislam@etro.vub.ac.be

Received 18 March 2009; Revised 20 November 2009; Accepted 23 December 2009

Recommended by Jan-Erik Mueller

\begin{abstract}
We present the design and construction of Frequency Selective Surface arrays composed of two subarrays of different slot lengths. We investigated their response variations with the variation of slot length differences of the elementary sub-arrays. Such nonhomogeneous arrays cannot be simulated with Computer Aided Design (CAD) programs because the boundary conditions are not fulfilled by the simulator. In infinite array simulation, the periodic boundary conditions are prescribed on the walls of the unit cell, whereas in the case of sub-arrays of unequal slot length such boundary conditions are not applicable. The CAD simulation of such combined array gives incorrect values of amplitude and phase responses. In this work, we investigate the characteristics of such complex arrays by using heuristic experimental approach. The results of the experimental approach demonstrate that the resultant reflection amplitude and phase of such complex array depend on the difference of slot lengths $(\Delta \mathrm{L})$ of the two sub-arrays.
\end{abstract}

Copyright (c) 2009 S. Islam et al. This is an open access article distributed under the Creative Commons Attribution License, which permits unrestricted use, distribution, and reproduction in any medium, provided the original work is properly cited.

\section{Introduction}

Frequency Selective Surfaces (FSSs) are passive electromagnetic filters formed by thin conducting elements on a dielectric substrate or periodic aperture elements in a conducting sheet [1]. FSSs have been widely employed for several years for the realization of antennas, radomes, passband and stop band filters and in many other devices. The FSS technologies became attractive due to their great versatility and simple manufacturing procedure [2-4]. More recently, the attention of the scientific community has been focused on applying FSS as an antenna system to reduce millimeter wave speckle. In this application a FSS array can be used as a phase diffuser system, phase delay element, digital phase modulator, mm-wave beam splitter and many other functions $[5,6]$. Such applications demand the exact characterization of complex arrays, especially arrays comprising subarrays of different slot lengths. Typically the analyses of FSSs and other planar periodic structures are carried out under the assumption that they are infinite in extent, even though the dimensions of practical structures are necessarily finite $[1,2,7]$. The methods of FSS simulation are based on a Floquet type representation of the fields in a unit cell, whose dimensions are typically in the order of a wavelength [7]. However, the conventional infinite array simulation techniques do not allow us to simulate the convenient extension to the more practical geometry, which comprises a large, but finite, number of subarrays. Except under the approximation that the edge effects are negligible, the analysis of the finite structure cannot be simplified by invoking periodicity, and it becomes necessary to work with a large number of unknowns, often in the order of a few thousands, in order to derive an accurate solution to the finite FSS problem [8].

In the design of large arrays, often smaller "building blocks" or subarrays are analyzed both by simulation and by measurement first. These blocks are usually too small to be analyzed by the infinite-array approach. The infinitearray approach cannot account for edge effects caused by the finiteness of the array and by the boundedness of the ground plane and dielectric layers [9]. With respect to the finiteness of the array, simulation results of unit cell are different from the measurement results of finite array that comprises the unit cells [10]. When mutual coupling, that is, 


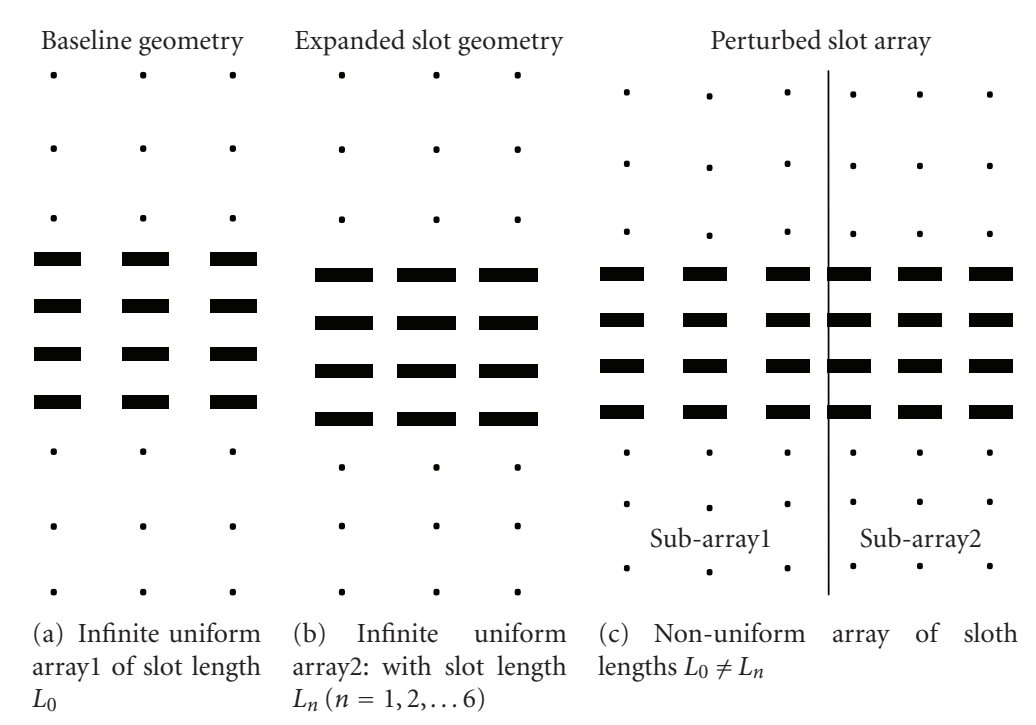

FIGURE 1: Two uniform and nonequal slot lengths infinite grounded FSS arrays ((a), (b)) and the compound arrays consisting of the two subarrays (c).

the interference effect between the elements is strong, there can be a considerable difference between the behavior of the edge elements and their infinite-array behavior [11]. In the array design, it can be possible to use the information obtained from the analysis of the subarrays in the analysis of the entire array. In this respect, it is important to know which information of the subarrays is essential for the description of the array behavior. Once this question is answered, the step towards a large array composed of subarrays can be made in an efficient way without the necessity of calculating again the subarrays information.

Arrays composed of subarrays of nonequal slot lengths cannot be analyzed by the infinite array approach due to the reasons mentioned above [9]. The expansion from finite array towards a complex infinite array could not be taken, because simulation of finite array (i.e., the elementary subarrays) requires too much computation time. Therefore, our focus remained on to investigate the effects of subarrays on the complex arrays. Analysis of complex FSS arrays is very much crucial for both designer and application engineers, because, CADs cannot deal with such finite arrays. In this work a heuristic experimental approach will be explored to develop design skills as long as the CADs programs do not allow for finite array simulation. The advantage of the experimental approach is that it can provide insight into the physics relevant to the array characteristics.

The FSS arrays under study consist of grounded slot FSS subarrays of different slot lengths (Figure 1). In this study we checked how the complex FSS array behaves with respect to its elementary subarrays. We also investigated how the finiteness of FSS arrays affects the experimental results and deviates from the infinite array simulations. The main objectives of the analysis approach are: to find characteristics of subarrays that are essential for describing the complex array behavior, for the development of an efficient design approach for finite FSS arrays on the basis of the single element characteristics. Our contribution in this paper is to the design process which specifically focused on the mutual coupling effect of the two nonequal slot length FSS subarrays. On the basis of experimental results, we focus on the characteristics that describe the qualitative behavior of the complex non-uniform FSS arrays. In particular, we find out characteristics of phase mixing behavior of different slot length FSS elementary subarrays which eventually will give the opportunity of nonuniform array characterization.

\section{Array Design Consideration}

The design methodology of the array is shown in Figure 1. The two infinite arrays with slot lengths $L_{0}$ (i.e., the baseline array or reference array) and $L_{n}$ are combined to make a compound array. The compound array is a non-periodic array as the slots of the subarrays are different. If the array is illuminated with the incident plane wave as indicated by the dotted circle of Figure 2, the electromagnetic (EM) fields emitted from the first subarray are incident upon the second subarray which needs not be operating or radiating itself. The second subarray can affect the fields from the first subarray and also absorbs and re-radiates them, so that electromagnetic fields from the second subarray are incident on the first subarray. The first subarray can then also absorb and re-radiate the fields from the second subarray. This process continues until a steady state is achieved. Hence, when an electromagnetic wave is applied to the first subarray, both the subarrays can radiate and the radiation from each subarray can be the result of several interactions. The nature of this interference, and therefore the total radiation, depends on the amplitude and the phase of the subarrays which are governed by the slot length of the individual subarrays. The array response will be different from the subarrays (infinite arrays of Figure 1) as the boundary conditions of the subarrays differ from the infinite periodic boundary 


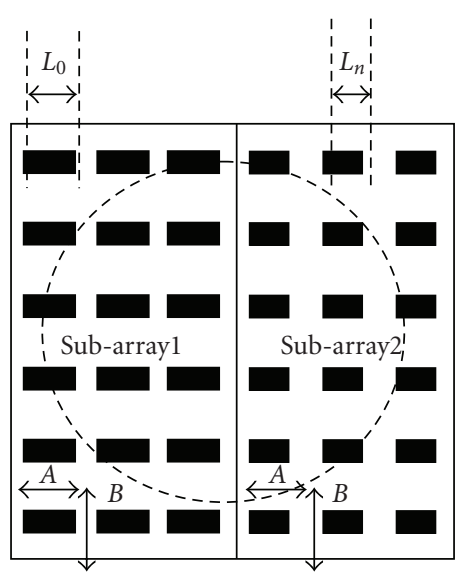

(a)

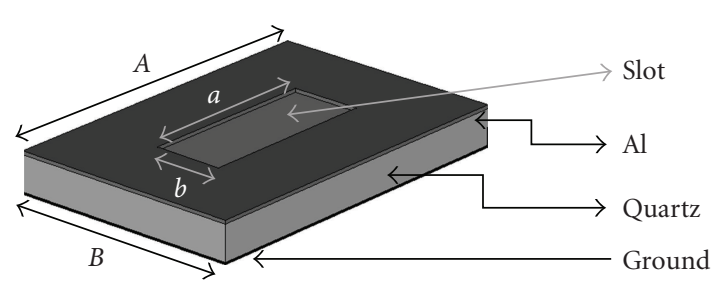

(b)

FIGURE 2: Non-uniform Grounded FSS array: (a) two subarrays with different slot lengths: dashed circle indicates the Millimeter Wave Vector Network Analyzer (MVNA) beam spot position and (b) constructional layout of unit cell configuration. The unit cell dimensions $A=B=1250 \mu \mathrm{m}=(0.7125) \lambda_{0} / 2\left[\lambda_{0}\right.$ is the free space wavelength at $85.5 \mathrm{GHz}]$.

applied in the infinite array simulation. First the baseline infinite array is simulated, designed and tested. Hence the baseline array is known. Note that the slot length of this baseline array is $L_{0}=811.5 \mu \mathrm{m}$ and the resonant frequency is measured at $85.5 \mathrm{GHz}$. To investigate the subarray behaviors, now six separate compound arrays are designed. Each array comprises of the fixed slot length baseline array as one of the subarrays and another subarray of slot length $L_{n}$ where $n=1,2,3,4$, and 5. The other slot lengths are $L_{1}=736$, $L_{2}=777 \mu \mathrm{m}, L_{3}=796 \mu \mathrm{m}, L_{4}=828 \mu \mathrm{m}, L_{5}=852 \mu \mathrm{m}$, and $L_{6}=920 \mu \mathrm{m}$, respectively. Hence the six arrays consist of subarrays of slot lengths $\left(L_{0} \& L_{1}\right),\left(L_{0} \& L_{2}\right),\left(L_{0} \& L_{3}\right),\left(L_{0}\right.$ $\left.\& L_{4}\right),\left(L_{0} \& L_{5}\right),\left(L_{0} \& L_{5}\right)$, respectively. We emphasize the main lines of the design process and subarray layout in the schematic representation of Figure 1.

The idea behind these arrays designs is to keep one of the subarray slot length fixed whereas the other slot length will be different. The slot lengths were obtained by means of microwave studio simulations applying the infinite boundary conditions. Taking into account that the slot length of the baseline array is $L_{0}=811.5 \mu \mathrm{m}$ the difference of slot lengths of the arrays are $\Delta L_{1}=75.5 \mu \mathrm{m}, \Delta L_{2}=34.5 \mu \mathrm{m}, \Delta L_{3}=$ $15.5 \mu \mathrm{m}, \Delta L_{4}=-16.5 \mu \mathrm{m}, \Delta L_{5}=-40.5 \mu \mathrm{m}$ and $\Delta L_{6}=$ $-108 \mu \mathrm{m}$. As mentioned in [9] the slot length of FSS is related to its phase value. Hence the variation in slot length means variation of resonant frequency and phase.

\section{Concept of Analysis}

A variety of approaches explicitly take the edge behavior into account for finite array simulation [12-14]. Based on the assumption that the edge behavior of an array is independent of the array size, the edge behavior of a large array is approximated in [15] by the edge behavior of a small array. More insight in edge phenomena is provided by the truncated Floquet-wave diffraction method [13, 14]. Within this method, an integral equation is solved for the fringe current, which describes the difference between the finite-array current and the associated infinite-array current. The fringe current is expanded into only a few basis functions defined on the entire array. The basis functions result from solutions of canonical problems, for example, determining (Green's) kernels for a planar semi-infinite array [15]. In [16], [17] the method is applied to rectangular arrays of open-ended waveguides with uniform and nonuniform amplitude excitation. Independently developed and strongly related to the truncated Floquet-wave diffraction method is the hybrid method described in [17]. The mutual admittance plays a key role in obtaining a low level of reflection. In fact, in this case, we are dealing with plane waves bouncing between two subarrays of different slot lengths. Hence, the infinite and semi-infinite (half-infiniteby-infinite) array solutions can be used to decompose the current on successive elements into a few standard distributions. The total reflection of the array comprised of two subarrays containing different load impedances as mentioned in [18]. When the self impedances of the two subarrays are equal there will be no grating lobes [2]. But when the self impedances of the two subarrays are not equal a simple frequency shift will be observed. This is the case we are dealing with this study. Furthermore, based on the general plane wave expansion, Hill has obtained a general expression for the mutual impedance [19]. So there is a need of experimental characterization of this type of arrays. Based on the experimental results we can conclude the exact phase response of a complex array.

\section{Fabrication}

The FSS arrays were fabricated on a grounded quartz substrate. The basic unit cell of the arrays is shown in Figure 2(a). The slots are etched in Aluminum on the quartz substrate (relative permittivity: 3.78, loss tangent: 0.0001, thickness: $800 \mu \mathrm{m})$. Slot lengths are obtained from the microwave studio simulations. The maximum number of allowable defects per square inch was 2 . The minimum feature size (i.e., critical dimension) we measured and controlled in the fabrication process is $5.0 \mu \mathrm{m}$. All the FSS arrays considered here are processed on the same wafer to avoid the relative processing error between arrays.

\section{Experimental Setup}

The measurements of the FSS arrays were carried out with the Millimeter Wave Vector Network Analyzer (MVNA) as shown in Figure 3. To get accurate results the MVNA 


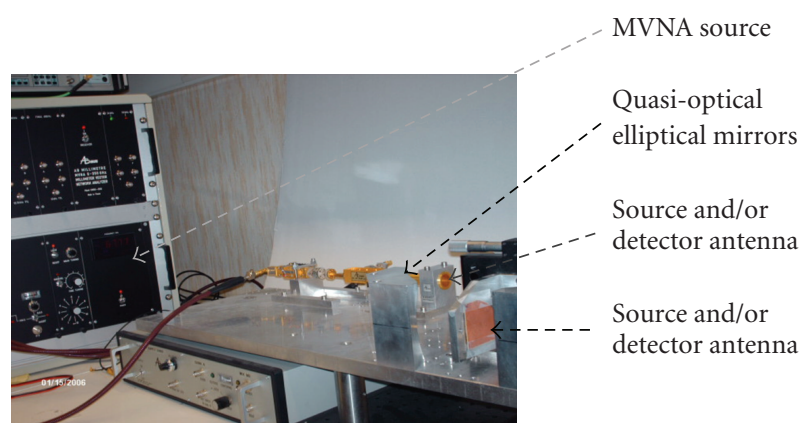

FIGURE 3: FSS measurement with Millimeter wave vector network analyzer (MVNA).

was properly calibrated. For transmission measurement a baseline correction or a response calibration which is done by first making a calibration measurement without any DUT while still recording the results. This is as if the test ports are directly connected to each other. Then later the measurement results with the DUT are divided by the recorded results of the calibration measurement.

The final outcome of this procedure is that the MVNA system can display the transmission response (both amplitude and phase) of the DUT. A suitable reference for the reflections of the DUT is easily obtained by making the calibration measurement with a short circuit as a calibration load. This follows from the fact that a short circuit in the test port of MVNA reflects quite precisely the entire test signal. Ideally the transmission and reflection responses would be directly related to the scattering parameters $S_{21}$ and $S_{11}$ of the DUT, respectively.

However, in practice, due to the limitations of the real world equipment, some case-dependent errors remain. The sources of the errors in the first case are, for example, the reflections between the DUT and the test ports of the network analyzer. In many cases isolators or attenuators in the test ports can be used to reduce these errors if necessary. In the second case, in practice, some errors will remain in the final measurement result and that is mostly due to the limitations of the equipment. In order to correct for these system errors and depending on the estimated accuracy requirements, a more complicated calibration technique can be used which is the calibration with loads other than only a short and open. Except the base calibration, it is also important in this measurement that the relative position of the FSSs on the holder should be the same for all the measurements. As the sizes of all the FSSs were same we confirmed the FSSs position by marking the antenna position on the MVNA holder. Several measurements are carried out for each array to characterize the FSS arrays accurately.

\section{Results}

The measured amplitudes and phases of FSS arrays are presented in Figure 4. It shows that the reflection amplitudes and phases of complex arrays depend on the difference of slot lengths of the two different sub arrays $(\Delta L)$.
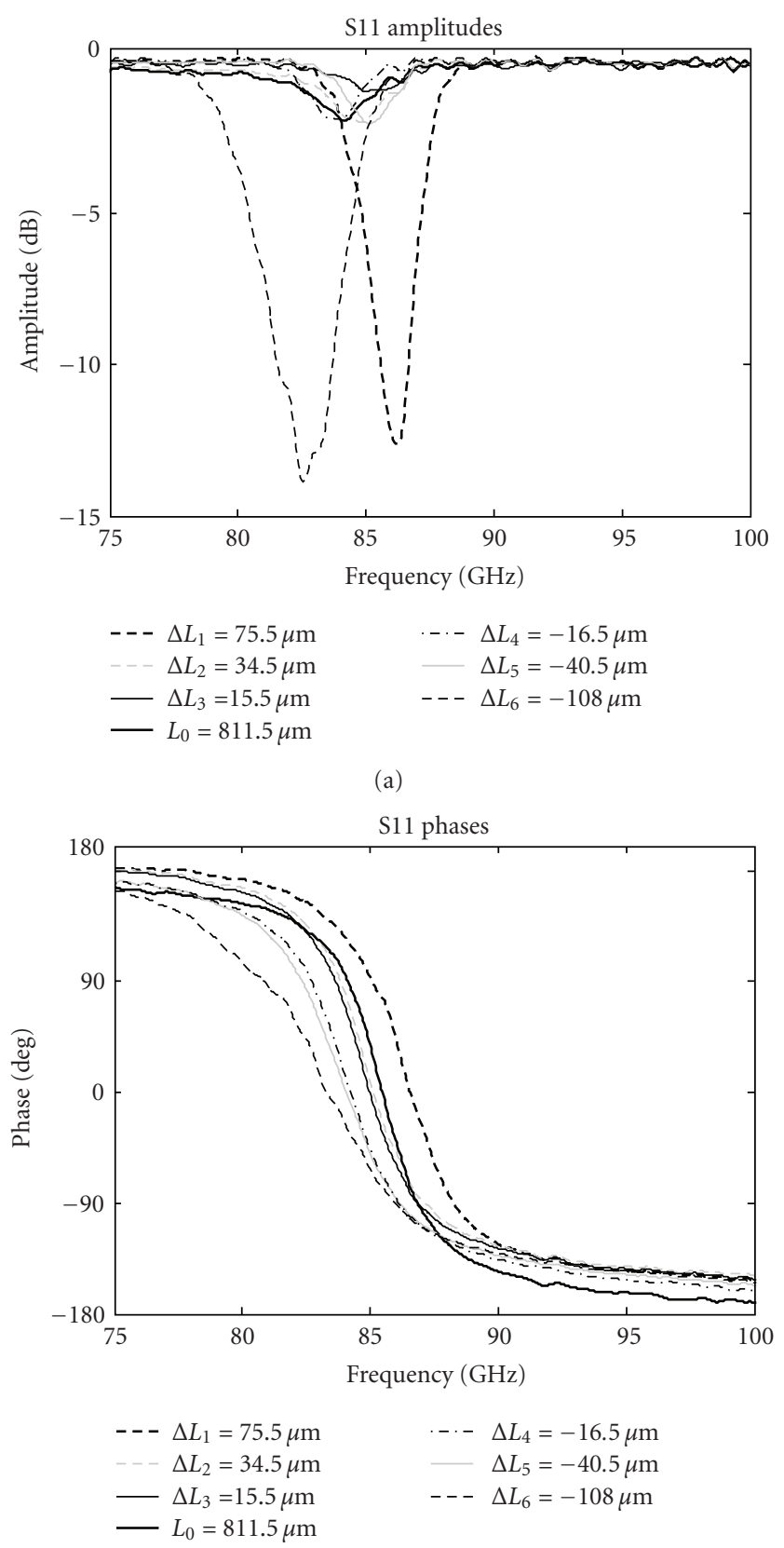

(b)

FIGURE 4: Measured reflection amplitudes and phases of nonuniform arrays of different slot lengths: (a) amplitudes and (b) phases.

In Figure 4 the solid line represent the amplitude and the phase response of a uniform baseline array of slot length $L_{0}=811.5 \mu \mathrm{m}$. The others are the complex arrays composed of subarrays of different $\Delta L$ values shown in the legend. In the legend " $+\Delta L$ " indicates that the array is composed with sub arrays of slot lengths $L_{0}$ and $L_{0}-\Delta L$ and " $-\Delta L$ " indicates the arrays are composed of subarrays of slot length $L_{0}$ and $L_{0}+\Delta L$. The results clearly shows the reflections for an oblique incidence which depends on special delay caused by the difference of slot lengths of the subarrays. Figure 4(b) 


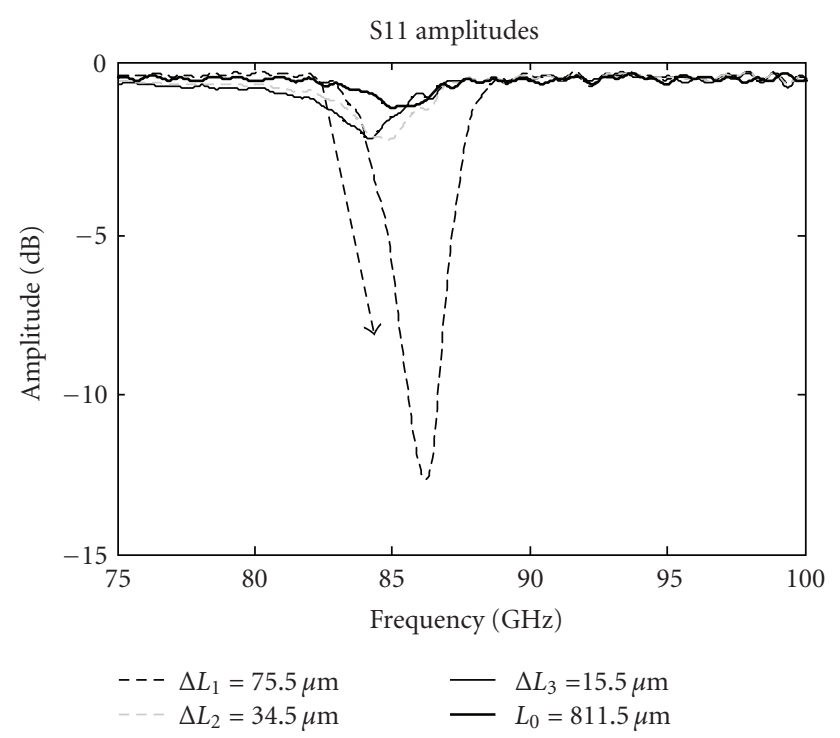

(a)

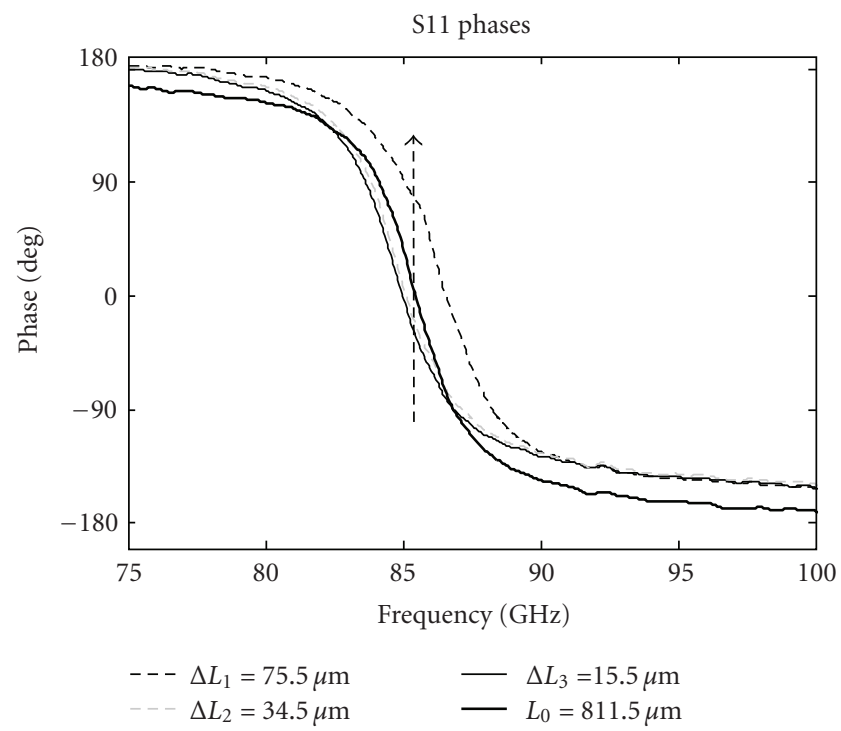

(b)

FIGURE 5: Indentation of FSSs amplitudes and phases change with $+\Delta L$ of subarray slot lengths.

shows that the phase delay between the subarrays depends on $\Delta L$.

Figures 5 and 6 represent the trends of amplitude and phase change with $+\Delta L$ and $-\Delta L$, respectively. Figure 5 presents that the reduction of subarray slot length shifts the resonant frequency to higher value and attenuation at resonance increases. When the slot length of the second subarray is reduced by $75.5 \mu \mathrm{m}$ the special phase delay between the subarrays is $135^{\circ}$ degree at $86.28 \mathrm{GHz}$ which causes destructive interference and we measured $-12.5 \mathrm{~dB}$ attenuation.

Figure 6 represents another set of experimental results where the each array is now composed of slot of length $L_{0}$ and $L_{0}+\Delta L$. Now the effective slot length is larger than

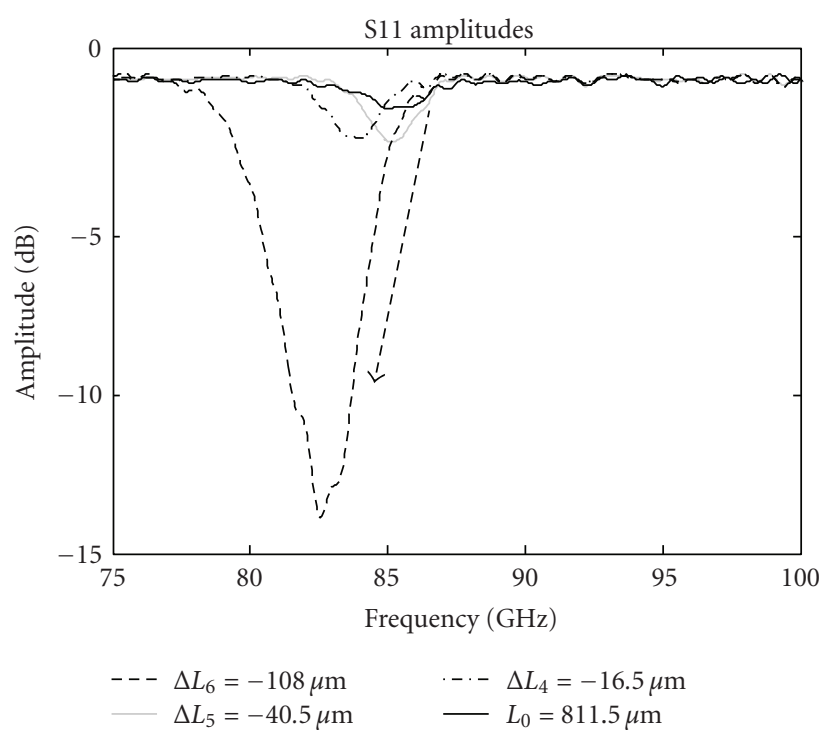

(a)

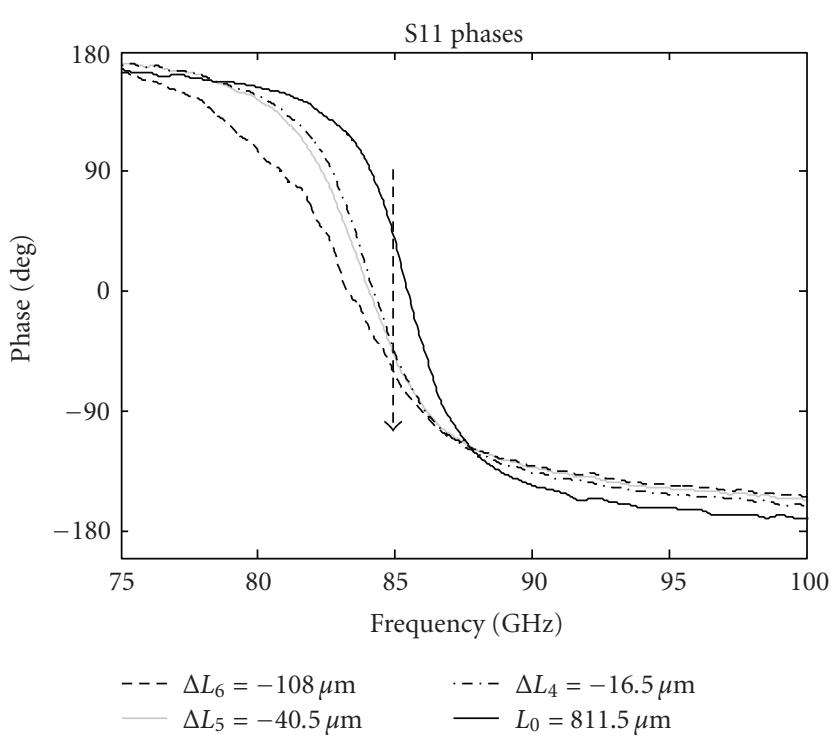

(b)

FIGURE 6: Indentation of FSSs amplitudes and phases change with $-\Delta L$ of subarray slot lengths.

that in the case of Figure 5. The resonance shifts towards lower frequency and attenuation at resonance also increases with the increment of $|\Delta L|$. We observed that when $\Delta L$ $=108 \mu \mathrm{m}$ a phase difference of $135^{\circ}$ at $82.86 \mathrm{GHz}$ causes the destructive interference and the reflection at resonance is $-13.86 \mathrm{~dB}$. As the reflection amplitudes and as well as the phases at resonance change with the value of $|\Delta L|$, it is necessary to investigate the relation of amplitudes at resonance with $\Delta L$. Figure 7 shows the plotted graph of $\triangle L$ versus attenuation at resonance for different arrays. It shows that the degree of mutual coupling between first and second array depends on their slot length difference $\Delta L$. It is shown that attenuation increases with the magnitude of slot length difference $(|\Delta L|)$ of the subarrays. For an oblique 


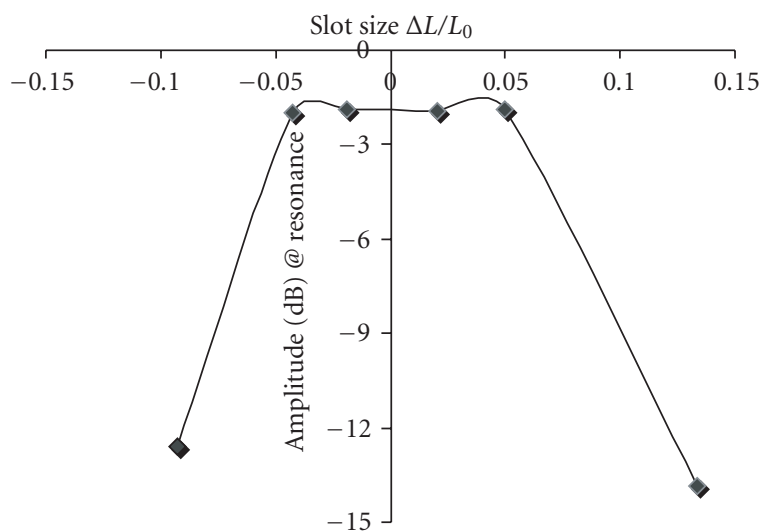

Figure 7: Measured reflection amplitudes versus slot size $\Delta L / L_{0}$ at the resonance.

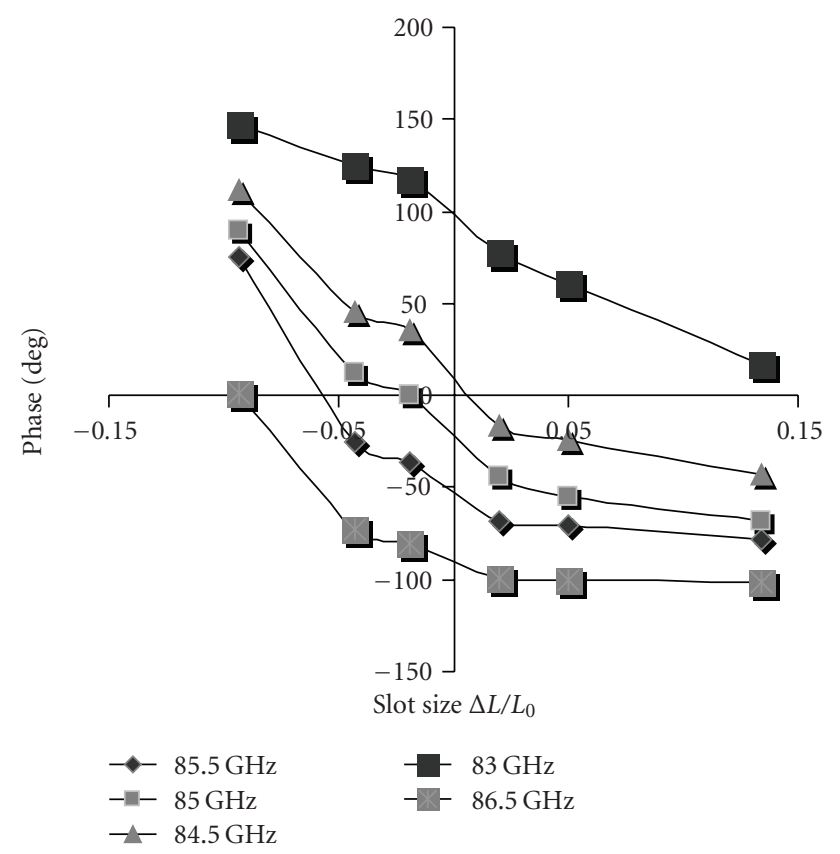

FIGURE 8: Measured reflection phase versus slot size $\Delta L / L_{0}$ of arrays at different frequencies.

incident angle if the delay between the two subarrays is an odd multiple of half wavelength, the reflected waves from the two subarrays are out of phase which causes destructive interference [20]. The interference between the subarrays increases with increasing value of $|\Delta L|$ which causes the end fire radiation. As a consequence the broad side radiation decreases.

Figure 8 shows the phase versus slot size $\Delta L / L_{0}$ of FSS arrays measured at different frequencies. We presented the phases versus $\Delta L$ at $83 \mathrm{GHz}, 84.5 \mathrm{GHz}, 85 \mathrm{GHz}, 85.5 \mathrm{GHz}$ and $86.5 \mathrm{GHz}$. Following these series of curves, it is possible to get the exact phase response of the complex array at the desired frequency if the slot length difference of the sub arrays is known. From Figures 7 and 8 it is possible to extract the exact phase and amplitude responses of a complex array if the value of $\Delta L / L_{0}$ of its subarrays is known. The phase value of the composite array at any frequency depends on the slot length difference of the subarrays.

\section{Conclusion}

This study aimed at getting insight into the effects of nonequal slot length subarrays of an array and the finite beam size in the reflection characteristics of arrays. As a basic antenna element we chose the grounded FSS. The experimental results clearly indicate the relation between the slot length difference $(\Delta L)$ of the subarrays and the composite array characteristics. We have presented a clear view in the order of magnitudes change in amplitude and phase when the slot length of the one subarrays changes while the other remains constant. The tendency of frequency shifting of the compound arrays compared to the baseline array indicates that the change in the slot length differences $( \pm \Delta L)$ of compound arrays has the similar effects on the phase curves when the slot length of a uniform array is changed. But the amplitude responses of the compound arrays have different scenarios as shown in Figure 4(a). The interference effect between the nonequal subarrays also depends on their slot length difference $(\Delta L)$ that is, the effect of mutual coupling between the subarrays is a function of the difference of their slot lengths. Moreover our investigation shows how the phase of an array composed of subarrays can be obtained if the slot length difference of the subarrays is known. Higher value of slot length difference $(\Delta L)$ can produce end fire radiation which may cause the broadside reflection very low even to zero when the phase difference of the subarrays of a compound array is $180^{\circ}$ [20].

\section{Acknowledgments}

This work was partially funded by the Vrije Universiteit Brussel (VUB-OZR) and the Flemish Institute for the encouragement of innovation in science and technology (IWT-SBO 231.011114).

\section{References}

[1] R. Kastner and R. Mittra, "Iterative analysis of finite-sized planar frequency selective surfaces with rectangular patches or perforations," IEEE Transactions on Antennas and Propagation, vol. 35, no. 4, pp. 372-377, 1987.

[2] C. C. Chen, "Transmission through a conducting screen perforated periodically with apertures," IEEE Transactions on Microwave Theory and Techniques, vol. 18, no. 9, pp. 627-632, 1970.

[3] C. C. Chen, "Diffraction of electromagnetic waves by a conducting screen perforated with circular holes," IEEE Transactions on Microwave Theory and Techniques, vol. 19, no. 5, pp. 475-481, 1971.

[4] S. W. Lee, "Scattering by dielectric-loaded screen," IEEE Transactions on Antennas and Propagation, vol. 19, no. 5, pp. 656-665, 1971.

[5] S. Islam, J. Stiens, G. Poesen, I. Jäger, and R. Vounckx, "Passive frequency selective surface array as a diffuser for destroying millimeter wave coherence," Active and Passive 
Electronic Components, vol. 2008, Article ID 391745, 6 pages, 2008.

[6] I. Jaeger, J. Stiens, L. Zhang, S. Islam, G. Koers, and R. Vounckx, "Comparison of speckle reduction diversity tools for active millimetre wave imaging," The Journal of the Optical Society of America A, vol. 25, no. 7, pp. 1716-1721, 2008.

[7] B. A. Munk, "Scattering from surface waves on finite FSS," IEEE Transaction on Antennas and Propagation, vol. 49, no. 12, 2001.

[8] C. H. Tsao and R. Mittra, "Spectraldomain analysis of frequency selective surfaces comprised of periodic arrays of cross dipoles and Jerusalem cross," IEEE Transaction on Antennas and Propagation, vol. 32, no. 5, pp. 478-486, 1984.

[9] D. Bekers, Finite antenna arrays: an eigencurrent approach, Ph.D. dissertation, Technische Universiteit Eindhoven, 2004.

[10] R. W. Scharstein, "Mutual coupling in a slotted phased array, infinite in E-plane and finite in H-plane," IEEE Transactions on Antennas and Propagation, vol. 38, no. 8, pp. 1186-1191, 1990.

[11] B. A. Munk, Frequency Selective Surfaces: Theory and Design, John Wiley \& Sons, New York, NY, USA, 2000.

[12] R. Mittra, C. H. Chan, and T. Cwik, "Techniques for analyzing frequency selective surfaces-a review," Proceedings of the IEEE, vol. 76, pp. 1593-1614, 1988.

[13] O. A. Civi, P. H. Pathak, H.-T. Chou, and P. Nepa, "A hybrid uniform geometrical theory of diffraction-moment method for efficient analysis of electromagnetic radiation/scattering from large finite planar arrays," Radio Science, vol. 35, no. 2, pp. 607-620, 2000.

[14] A. Neto, S. Maci, G. Vecchi, and M. Sabbadini, "A truncated Floquet wave diffraction method for the full-wave analysis of large phased arrays-part II: generalization to 3-D cases," IEEE Transactions on Antennas and Propagation, vol. 48, no. 4, pp. 601-611, 2000.

[15] A. Polemi, A. Toccafondi, and S. Maci, "High-frequency green's function for a semi-infinite array of electric dipoles on a grounded slab-part i: formulation," IEEE Transactions on Antennas and Propagation, vol. 49, no. 12, pp. 1667-1677, 2001.

[16] A. Cucini, M. Albani, and S. Maci, "Truncated floquet wave full-wave $(\mathrm{T}(\mathrm{FW}) 2$ ) analysis of large periodic arrays of rectangular waveguides," IEEE Transactions on Antennas and Propagation, vol. 51, no. 6, pp. 1373-1385, 2003.

[17] A. Neto, S. Maci, G. Vecchi, and M. Sabbadini, "A truncated Floquet wave diffraction method for the full wave analysis of large phased arrays-part I: basic principles and 2-D cases," IEEE Transactions on Antennas and Propagation, vol. 48, no. 4, pp. 594-600, 2000

[18] B. A. Munk, Finite Antenna Arrays and FSS, John Wiley \& Sons, New York, NY, USA, 2003.

[19] R. A. Hill, The design of a dual band frequency selective surface and the effect of perturbing the elements and the inter-element spacing, Ph.D. dissertation, Ohio State University, Department of Electrical Engineering, Columbus, Ohio, USA, 1991.

[20] S. Islam, J. Stiens, G. Poesen, I. Jaeger, and R. Vounckx, "Grounded frequency selective surface array characterization for quasi optical phase shifter at W-band," International Journal on Information and Communication Technologies (IJICT), vol. 2, pp. 240-244, 2009. 

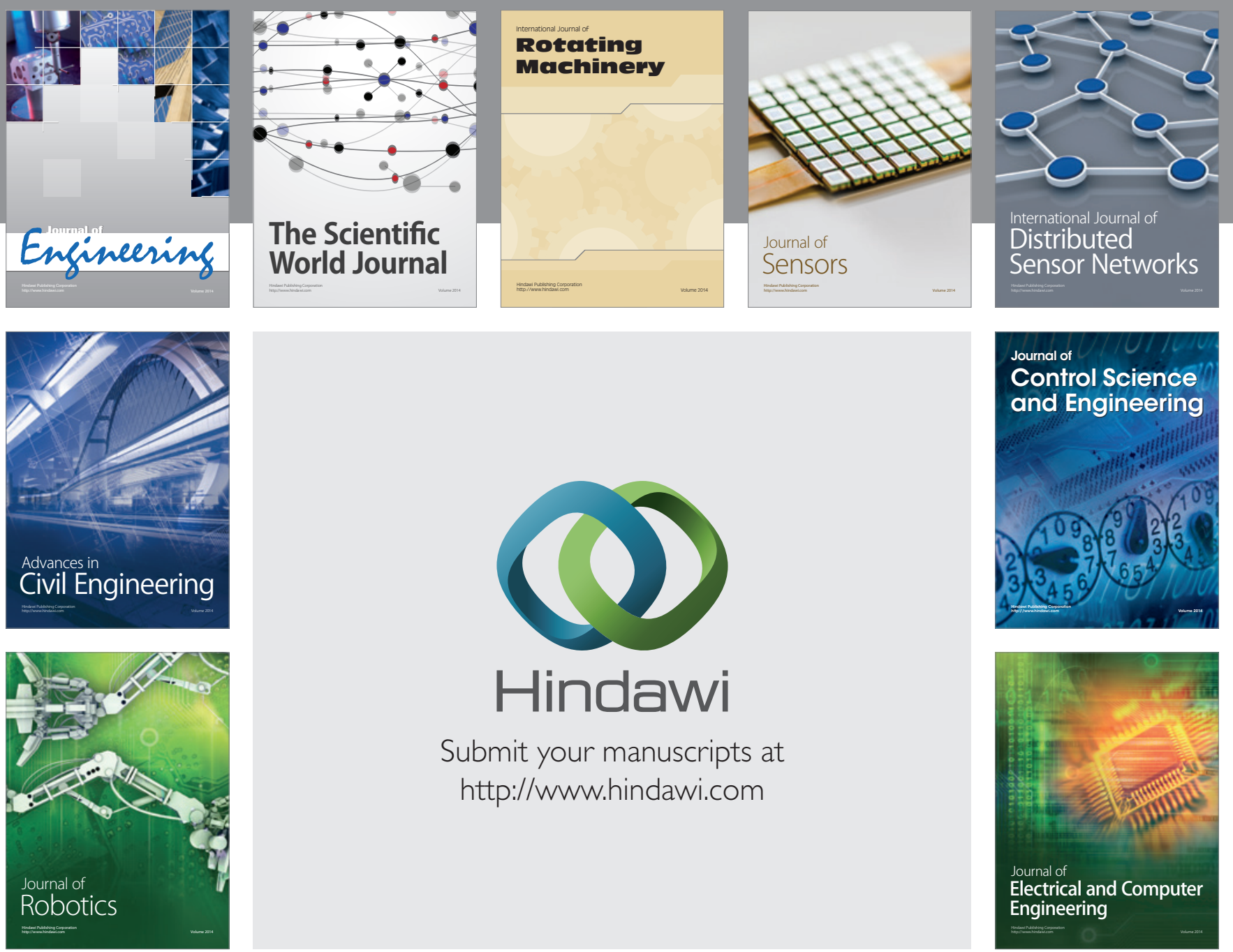

Submit your manuscripts at

http://www.hindawi.com
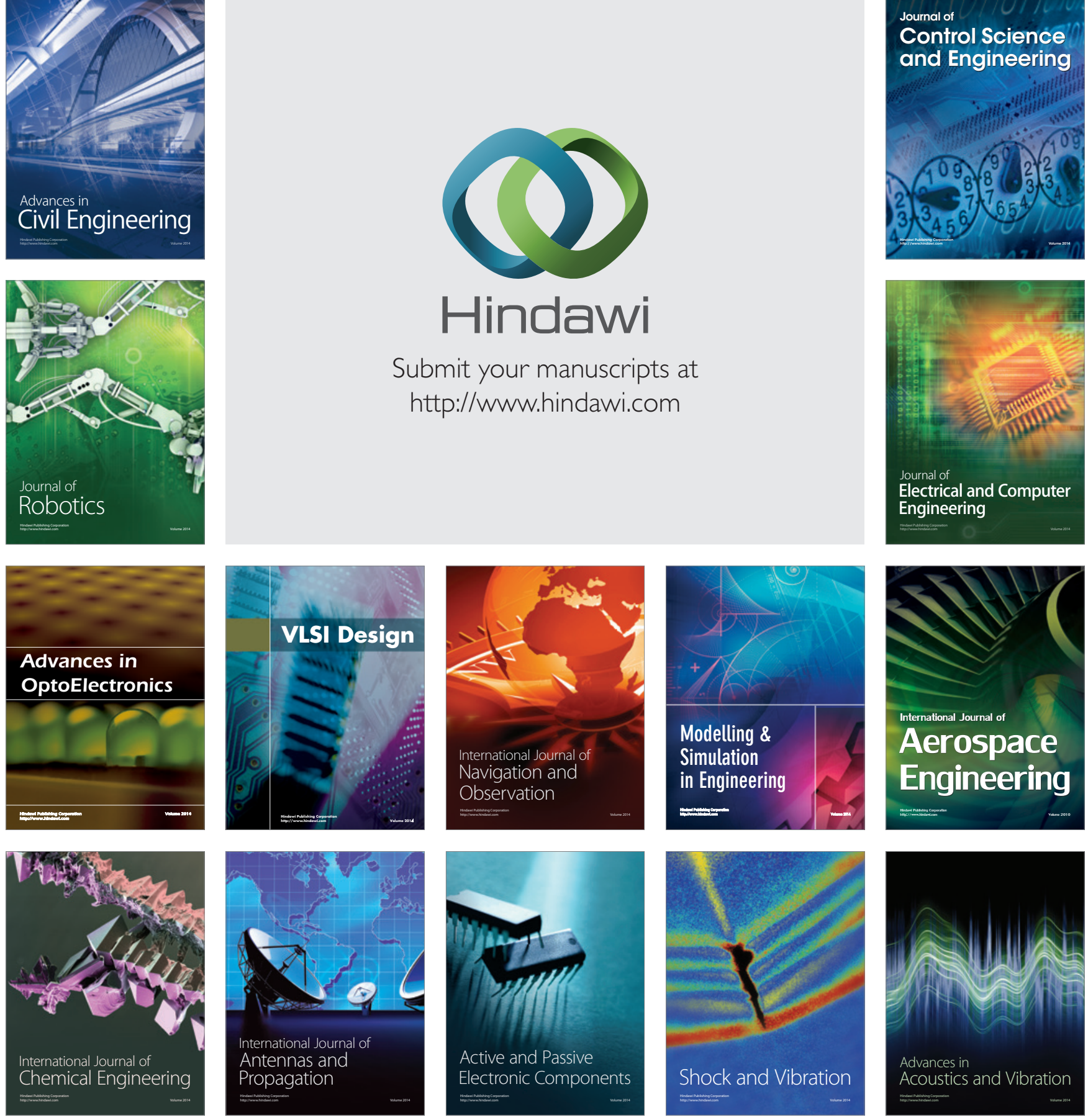